MATEC Web of Conferences 13, 01002 (2014)

DOI: $10.1051 /$ matecconf/ 20141301002

(C) Owned by the authors, published by EDP Sciences, 2014

\title{
Towards an Improved Flow Rate Estimation Method
}

\author{
Muhammad Hazim bin Suffian ${ }^{1}$ and Mark Ovinis ${ }^{1, a}$ \\ ${ }^{1}$ Mechanical Engineering Department, Universiti Teknologi PETRONAS, 31750 Tronoh, Malaysia
}

\begin{abstract}
This paper presents the development of a technique for estimating flow rate. The intended application is to estimate the volume of oil spilled from a ruptured subsea pipeline, with improved accuracy over existing methods. In this work, two novel methods to estimate flow rate with high accuracy were considered. The first involves the use of 3D cameras to estimate volumetric flow rate and the other uses a 2D camera to estimate the flow velocity. Related works including 3D reconstruction, optical flow and the experimental setup are described. The 2D camera approach produced good results, with an error of $2 \%-9 \%$. On the other hand, the use of $3 \mathrm{D}$ cameras needs further work, as the cameras are unable to detect the flow. Future work for this approach will be focused on overcoming this limitation and developing the method further.
\end{abstract}

\section{Introduction}

The Deepwater Oil Disaster that occurred on 22nd April 2010 is the largest accidental spill [1]. It took the operator, BP and the US government 87 days to seal the oil well. Numerous efforts had been taken ranging from a specially built dome to junks and mud to control the leak. However, one issue still remained uncertain, which was the estimated volume of oil spilled into the ocean, as penalties imposed are based on the amount oil leaked. The most accurate method to date is a standard deviation of $2.6 \%$ by Crone [2]. As oil spills are typically in millions of barrels, an inaccurate estimate will result in a substantial error. Thus, there is a need to accurately estimate the total amount of oil leaked. In this work, a more accurate flow estimation technique is described. The development of this new method will enable the volume of oil spilled from a ruptured subsea pipeline to be estimated with higher accuracy. It may also have the potential to be applied to other flow rate estimation. Two types of technique were used to estimate the volume of oil spilled in the Deepwater Oil Disaster, those based on satellite imagery and those based on video of the flow [3]. The estimated flow rate varies quite significantly for each method. Table 1 summarizes the different flow rate estimation techniques, the estimated flow rates and their limitations. Estimates based on video of the flow are generally more accurate than estimates based on satellite imagery.

This is an Open Access article distributed under the terms of the Creative Commons Attribution License 2.0, which permits unrestricted use, distribution, and reproduction in any medium, provided the original work is properly cited. 
Table 1. Flow rate estimation methods and their limitations

\begin{tabular}{|l|c|c|l|}
\hline \multicolumn{1}{|c|}{ Method } & Author & $\begin{array}{c}\text { Estimated Flow } \\
\text { Rate (bbls/day) }\end{array}$ & \multicolumn{1}{|c|}{ Limitation } \\
\hline $\begin{array}{l}\text { Satellite imagery without } \\
\text { Bonn Convention Protocol }\end{array}$ & $\begin{array}{c}\text { John Amos } \\
{[4]}\end{array}$ & $5,000-20,000$ & $\begin{array}{l}\text { Assumption of no oil burned and } \\
\text { evaporated }\end{array}$ \\
\hline $\begin{array}{l}\text { Satellite imagery with } \\
\text { Bonn Convention Protocol }\end{array}$ & $\begin{array}{c}\text { Ian } \\
\text { Mcdonald [5] }\end{array}$ & 26,500 & $\begin{array}{l}\text { Bonn Convention not recommended for } \\
\text { analysing large spills [4] }\end{array}$ \\
\hline $\begin{array}{l}\text { Optical Plume } \\
\text { Velocimetry }\end{array}$ & $\begin{array}{c}\text { Timothy } \\
\text { Crone [2] }\end{array}$ & $50,000-100,000$ & $\begin{array}{l}\text { Poor imaging system } \\
\text { L Low speed (fps) } \\
\text { • Low resolution }\end{array}$ \\
\hline $\begin{array}{l}\text { Angle of flow and the rate } \\
\text { of flow }\end{array}$ & $\begin{array}{c}\text { Eugene } \\
\text { Chiang }\end{array}$ & $20,000-100,000$ & $\begin{array}{l}\text { Assumption on the percentage of oil from } \\
\text { the flow. } \\
\text { No information inside the flow. }\end{array}$ \\
\hline $\begin{array}{l}\text { Particle Image } \\
\text { Velocimetry }\end{array}$ & $\begin{array}{c}\text { Steven } \\
\text { Wereley [7] }\end{array}$ & $72,129( \pm 20 \%)$ & \multicolumn{2}{|l}{} \\
\hline
\end{tabular}

\subsection{Flow Rate Estimation}

Flow rate is defined as the volume of fluid that flows past a given cross sectional area per second. There are two general methods to estimate flow rate, volume based and velocity based. One way to estimate flow rate is to determine the rate of volume change over time. The volume of fluid can be estimated based on a $3 \mathrm{D}$ reconstruction of a flow [10]. The flow rate can be estimated per Equation 1:

$$
Q=d V / d t
$$

where $Q$ is the flow rate $[\mathrm{cm} 3 / \mathrm{s}], V$ is the volume $\left[\mathrm{cm}^{3}\right]$ and $t$ is the time $[\mathrm{s}]$. For a steady and incompressible fluid flow involving only one stream of a specific fluid flowing through a control volume [6], the flow rate is given by Equation 2:

$$
Q=v A
$$

where $v$ is the velocity $\left[\mathrm{cm}^{2}\right]$ and $A$ is the cross sectional area $\left[\mathrm{cm}^{2}\right]$.

\subsection{Related Works}

Methods based on video of the flow are more accurate compared to those based on satellite imagery, as evident by the results obtained from work of Crone, Chiang and Wereley. Recent work on video analysis to estimate flow rate include that by Emalisa [8] and Khairi [9], who did some preliminary work on flow rate estimation. Khairi used a Kinect for 3D reconstruction, with the aim of eventually using this technique for flow rate estimation. Kinect is an optical camera equipped with a depth sensor, and is able to return 3D coordinates of an object. An image processor uses an infrared pattern [11] to calculate the object depth. Table 2 shows a summary of their work.

Table 2. Emalisa and Khairi's flow estimation method

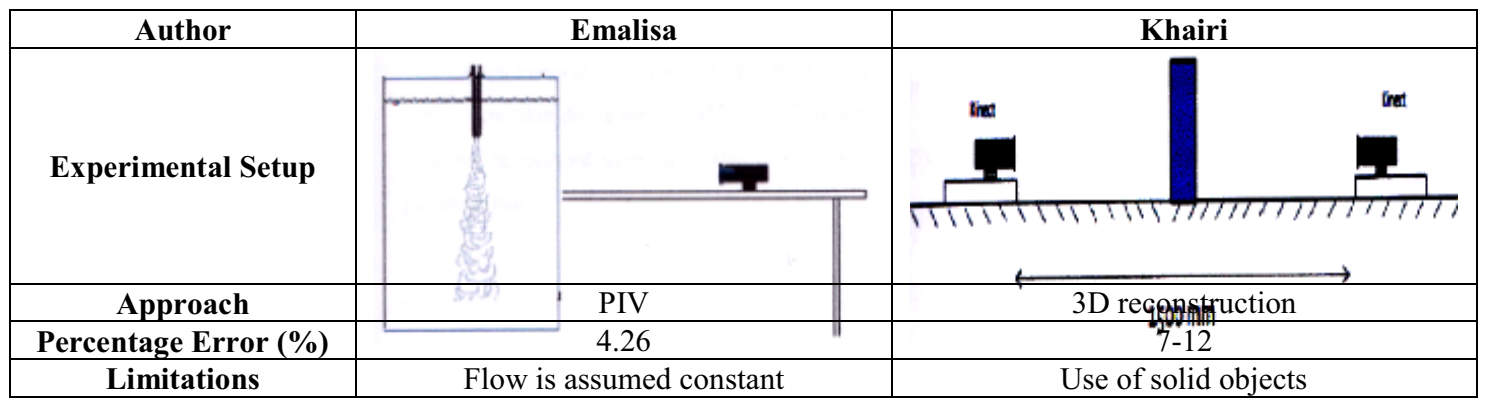




\section{Methodology}

\subsection{Experimental Setup}

Crone's experimental setup (which was used to simulate an oil spill from a pipeline), as shown in Figure 1, was replicated. In this work, three Kinects place $120^{\circ}$ apart were used in place of the single $2 \mathrm{D}$ video camera, to facilitate $3 \mathrm{D}$ reconstruction of the flow.

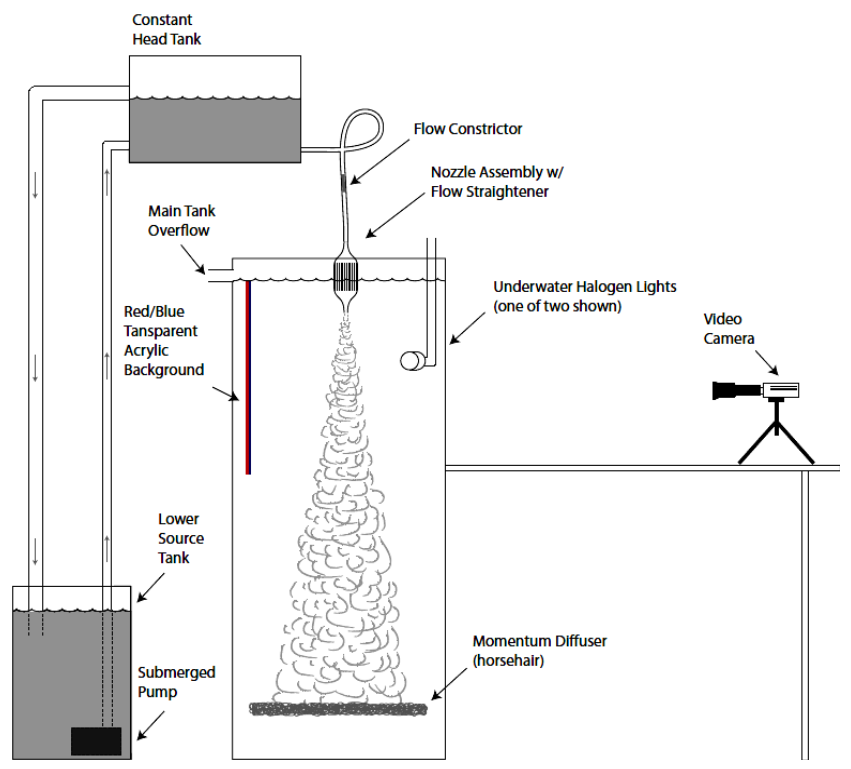

Figure 1. Crone's experimental setup [2]

\subsection{Volume Estimation}

For volume estimation of a flow, 3D reconstruction based on point clouds from three Kinects was performed. A transformation matrix and Iterative Closest Point that relates the point cloud of one Kinect to another were used. The volume of a basic alpha shape [7] for the combined point cloud is the volume of the flow. Alpha shapes are a generalization of the convex hull of a point set, essentially the volume bounded by a set of points.

\subsection{Optical Flow}

An alternative technique based on optical flow was also implemented to estimate the velocity of the fluid flow. Optical flow is the apparent motion of brightness patterns in an image corresponding to the motion field [12]. One of the most common optical flow methods is that by Lucas and Kanade [13]. The algorithm attempts to find an optimal value for a disparity vector, $h$, which represents an object's displacement (pixel/frame) between successive images [14]. Their method presents a desirable level of flow accuracy which is capable of distinguishing regions varying in activity level [14]. The velocity of a fluid flow can then be estimated as given in Equation 3:

$$
v=h . F P S . k
$$


where $v$ is velocity [cm/s], $h$ is pixel displacement [pixel/frame], FPS is the camera speed [frame/s] and $k$ is calibration constant $(\mathrm{cm} / \mathrm{pixel})$. The assumptions made in estimating velocity were that the flow was assumed to be in a pipe (conservation of mass) with a circular cross section with a region of interest near the nozzle chosen.

\section{Results and discussion}

Videos of flow from the experimental setup as shown in Figure 1 had been successfully captured. Figure 2 shows a sample video frame with an average camera speed, $F P S=19 \mathrm{frame} / \mathrm{s}$ and with a calibration constant, $k=0.1319 \mathrm{~cm} /$ pixel.

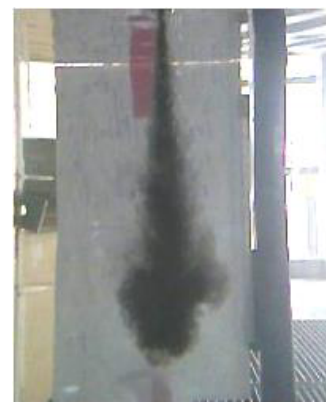

Figure 2. Video of flow

Unfortunately, the flow cannot be detected by the infrared laser patterns from the Kinect. As such, depth values and hence 3D reconstruction of the flow could not be obtained. To further develop this method, a solid object was used in place of the flow. The 3D reconstruction of the solid object, by merging information obtained from three Kinects, is shown in Figure 3.
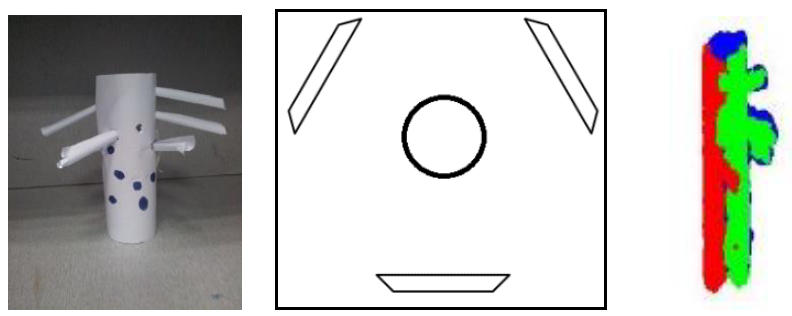

Figure 3. (a) Solid object used (b) Arrangement of the three Kinects (c) 3D reconstruction

The volume of the reconstructed 3D object, as shown in Figure 3, was estimated with alpha shapes. The estimated volume was $1018.4 \mathrm{~cm}^{3}$ while the actual volume was estimated to be 640.96 $\mathrm{cm}^{3}$, an error of $58.89 \%$. The error is mainly because estimating volume for a point cloud is inaccurate. The estimated flow rate based on optical flow, as shown in Table 2, yielded better results.

Table 2. Flow rate estimation based on optical flow

\begin{tabular}{|c|c|c|}
\hline Parameters & Flow 1 & Flow 2 \\
\hline Pixel displacement, $h[\mathrm{pixel} /$ frame] & 2.0677 & 2.1276 \\
\hline Velocity, $v[\mathrm{~cm} / \mathrm{s}]$ & 5.1819 & 5.3320 \\
\hline Diameter $[\mathrm{cm}]$ & 2.9 & 2.5 \\
\hline Area, $A\left[\mathrm{~cm}^{2}\right]$ & 6.6061 & 4.9094 \\
\hline Estimated flow rate $\left[\mathrm{cm}^{3} / \mathrm{s}\right]$ & 34.2321 & 26.1769 \\
\hline Actual flow rate $\left[\mathrm{cm}^{3} / \mathrm{s}\right]$ & 33.5189 & 24.0132 \\
\hline Percentage Error $[\%]$ & 2.08 & 9.01 \\
\hline
\end{tabular}


The error for flow 2 is higher. The probable cause of the higher error is because of the presence of bubbles in the flow, which reduced the accuracy of the estimation. As such, the technique may not be appropriate for flow where the amount of bubbles is significant.

\section{Conclusions}

Two approaches were considered to estimate flow rate better. The 3D approach needs further work as Kinect is unable to detect depth underwater as originally thought. Future work for this approach can be focused on overcoming this limitation and on developing more accurate techniques on volume estimation from a point cloud. A possible solution would be the use of a 3D camera based on optical images rather than infrared patterns. The optical flow approach produced a low error of $2 \%-9 \%$. Future work should focus on improving the technique so that it is robust in the presence of bubbles in the flow. As a conclusion, a more accurate method in flow rate estimation was developed.

\section{References}

1. Moss, Laura. "The 13 Largest Oil Spills in History." Mothernature Network. N.p., (16 July 2010). "http://www.mnn.com/earth-matters/wilderness-resources/stories/the-13-largest-oil-spills-inhistory $>$ ".

2. Crone, T. J., McDuff, R. E., \& Wilcock, W. S., Experiments in fluids. 45(5), (2008) 899-915.

3. Prepared by CRS; estimates and timeline from National Commission on the BP Deepwater Horizon Oil Spill and Offshore Drilling, The Amount and Fate of Oil, Staff Working Paper, (October 6, 2010). "http://www.oilspillcommission.gov"

4. John. "Gulf Oil Spill Rate Must Be Much Higher Than Stated - 6 Million Gallons So Far?" Web log post. Skytruth. N.p., 27 Apr. 2010. "http://blog.skytruth.org/2010/04/gulf-oil-spill-rate-mustbe-much-higher.html"

5. Gillis, Justin. "Size of Oil Spill Underestimated, Scientists Say." The New York Times. N.p., 13 May 2010. "http://www.nytimes.com/2010/05/14/us/14oil.html?_r=1\&"

6. Munson, Bruce Roy. Fundamentals of Fluid Mechanics, Sixth Edition. 6th ed. Hoboken, NJ: Wiley, 195 (2009)

7. Edelsbrunner, H., \& Mücke, E. P., ACM Transactions on Graphics (TOG), 13(1), 43-72 (1994)

8. Kamsani,E. Development of an automated flow rate estimation method. Dissertation, Universiti Teknologi PETRONAS, Malaysia. (2012)

9. Rahim,K. Development of a 3D automated flow estimation technique. Dissertation, Universiti Teknologi PETRONAS, Malaysia. (2012)

10. Stelldinger, P., \& Latecki, L. J., In Proc. IEEE Int'l Conf. Image Analysis, (2006)

11. Andersen, M., Jensen, T., Lisouski, P., Mortensen, A., Hansen, M., Gregersen, T., \& Ahrendt, P., Technical report ECETR-6, Department of Engineering, Aarhus University Denmark. (2012)

12. Robyn Owens, Optical flow. "http://homepages.inf.ed.ac.uk/rbf/CVonline/LOCAL_COPIES/OWENS/LECT12/node4.html"

13. Lucas, B. D., \& Kanade, T., In IJCA, 81, 674-679 (1981)

14. Bruhn, A., Weickert, J., \& Schnörr, C., Lucas/Kanade meets Horn/Schunck: International Journal of Computer Vision, 61(3), 211-231 (2005) 